\title{
Idiopathic Multiple Impacted Teeth: A Case Report
}

\author{
Dhafer Al Rakaha, b, Hessa Al Muhanna ${ }^{\text {a }}$, Saad El Ghazali ${ }^{\mathrm{a}}$
}

\begin{abstract}
Multiple impacted teeth are related to syndromes, hormonal and metabolic disorders. But in some cases it is not associated with any syndrome, and may be affected by infection, cyst and trauma. Here we present a case of 34-year-old male with multiple missing teeth. Radiographic interpretation revealed multiple impacted permanent teeth. Medical and family history with physical examination was not suggestive of any syndromes. Idiopathic multiple impacted teeth are suggested to be the possible diagnosis. The objective is to increase awareness of such cases in the absence of hereditary, genetic and metabolic factors.
\end{abstract}

Keywords: Multiple impacted teeth; Missing teeth; Idiopathic

\section{Introduction}

The teeth eruption is defined as the occlusal or axial movement of the teeth from its developmental position within the jaw to its functional position in the occlusal plane [1]. The impacted teeth are known as the teeth which cease to erupt before emergence to the oral cavity. Insufficient space, early loss of primary teeth with eventual closure of space, crowding of arches and rotation of tooth buds are the common and important reasons attributed to delayed eruption. Excessive fibrous tissue over an erupting tooth is also one of the most common causes, with eruption cysts being relatively rare [1].

Complete lack of eruptive force can be the reason for unerupted tooth when the normal number of teeth is present radiographically, although this is debatable, since few workers have debunked this claim [2, 3]. Syndromes, metabolic, and hormonal disorders are also other causes of multiple impacted permanent teeth. Finally crowding and rotation of tooth buds can also lead to impacted teeth [4].

The objective of this study is to increase the importance and awareness of cases of multiple unerupted (impacted) teeth in which multiple etiologies need to be discussed and investigated.

Manuscript submitted December 8, 2017, accepted December 18, 2017

aDepartment of Dentistry, King Saud Medical City, Riyadh, Saudi Arabia ${ }^{b}$ Corresponding Author: Dhafer Al Rakah, Department of Dentistry, King Saud Medical City, Riyadh, Saudi Arabia. Email: dr-dhafer@hotmail.com

doi: https://doi.org/10.14740/jmc2986e

\section{Case Report}

A 34-year-old Saudi male patient reported with a chief complaint of missing teeth and compromised aesthetics. The patient was of normal build with no previous medical history. Dentally, all of the maxillary erupted teeth were treated endodontically. He had no relevant family history and this was his first prosthodontics visit. On further enquiry the patient revealed that he had loss of deciduous teeth with no eruption of permanent teeth. On examination, a total of 13 teeth were present in the patient's oral cavity exhibited with fluorosis and arrested caries (Figs. 1, 2). The patient had multiple impacted permanent teeth with only the permanent maxillary central and lateral incisors, mandibular central and lateral incisors, right maxillary second molar and second premolar, left maxillary second premolar, and mandibular first molars are present. The incisal length of the maxillary central incisors appeared reduced, reduced vertical dimension of occlusion, pseudo class III malocclusion and the patient had a deep palatal vault. When orthopantomogram (OPG) was performed, it demonstrated impacted, unerupted, and missing mandibular third molars (Fig. 3). Skull X-rays did not reveal other anomalies (Fig. 4), and the chest X-ray was unremarkable (Fig. 5).

\section{Discussion}

Epidemiological studies have reported dental impactions to affect $25 \%$ to $50 \%$ of the population [5]. Multiple impactions

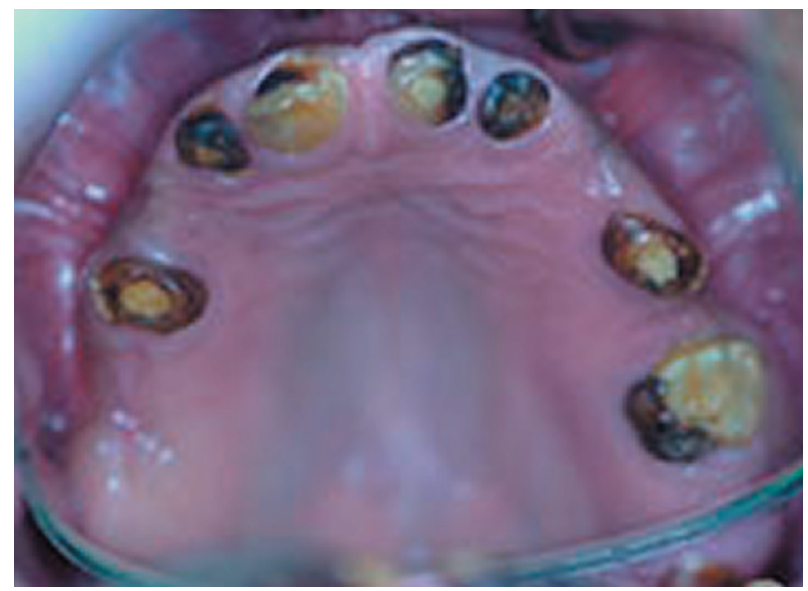

Figure 1. Partially edentulous maxilla with remained carious and rotated teeth and deep palatal vault. 


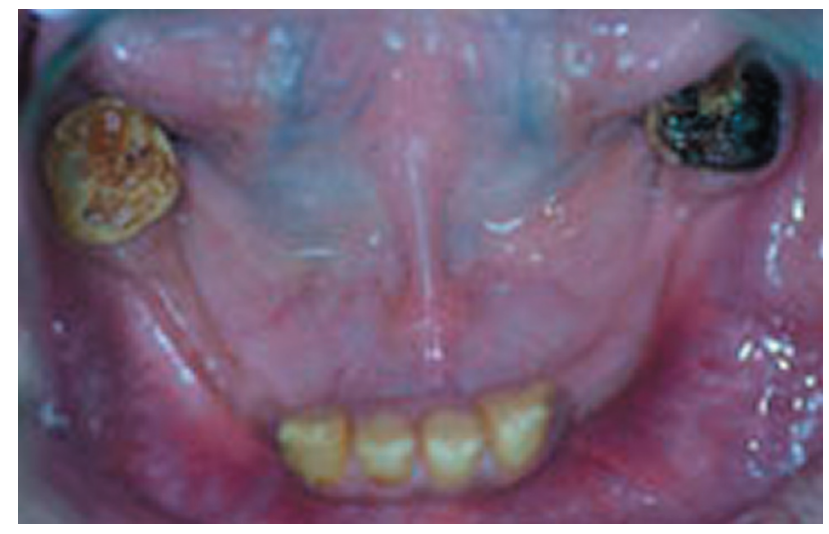

Figure 2. Partially edentulous mandible with remained carious teeth.

are seen rarely and are usually associated with systemic conditions. Guided eruption of many teeth with the help of coordinated multidisciplinary management is needed for patients with multiple impactions [6].

There are numerous eruption-regulating molecules having similar and overlapping functions, which ensure that even the absence of a single factor does not interrupt the event of eruption [1]. But defect in some genes may be responsible for this condition. EGF, EGF-R, CSF-1, CSF-1R, IL-1, IL-1R, c-Fos, NFB, MCP-1, TGF- $\beta 1$, PTHrP, Cbfa-1 (now called Runx2), OPG, and RANK/RANKL are the major tooth eruption molecules. Stellate reticulum is the site for few molecules with the majority of the eruption molecules residing in the dental follicle [6].

While most eruption defects are part of a genetic syndrome, they can also be non-familial, as in the case in primary failure of eruption (PFE). In PFE localized failure of eruption of permanent teeth exists with no other systemic involvement.

Hence, it is likely that genes like CSF-1, NFB, and c-fos are the genes responsible for the eruption defect [3].

The causes of tooth impaction are divided into general-

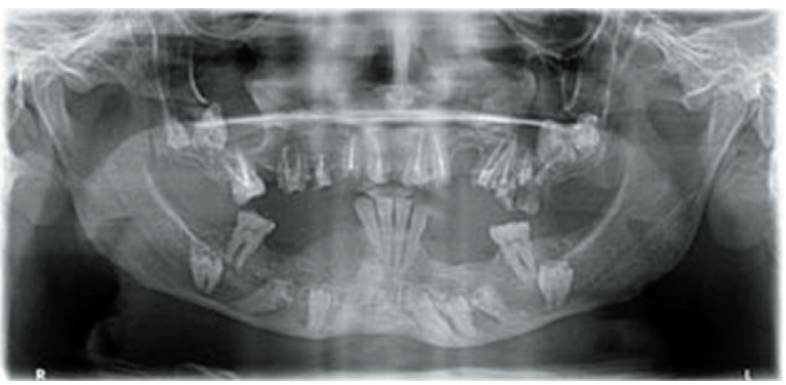

Figure 3. The OPG revealed the presence of impacted, unerupted, and missing mandibular third molars. Few teeth showed enamel hypoplasia and calcifications in the pulp space.

ized and localized. Cleidocranial dysplasia is the most common syndromal cause of tooth impaction. Common reasons like lack of space, prolonged retention or early loss of deciduous tooth, abnormal position of tooth bud, presence of alveolar cleft, ankylosis, cystic or neoplastic formation, alveolar or dental trauma, and dilacerations of teeth falling into the localized category $[7,8]$.

Multiple impacted teeth are usually present in syndromes. The differential diagnoses in case of syndromes are cleidocranial dysostosis, Gardner's syndrome, Gorlin-Sedano syndrome, and Yunis-Varon Syndrome [9, 10].

In the case of cleidocranial dysplasia the patients usually exhibit high-arched palate with prolonged retention of the deciduous teeth leading to subsequent delay in eruption of the permanent teeth. Though both the intraoral features were present in our case, the abnormalities of skull and shoulder girdle along with multiple supernumerary impacted teeth were absent. Gardner's syndrome that also presents with multiple impacted teeth was ruled out as associated signs like multiple epidermoid cysts and other lesions were absent. Gorlin-Sedano syndrome represents as short hands, foot bones with short and straight collar bone along with multiple impacted teeth
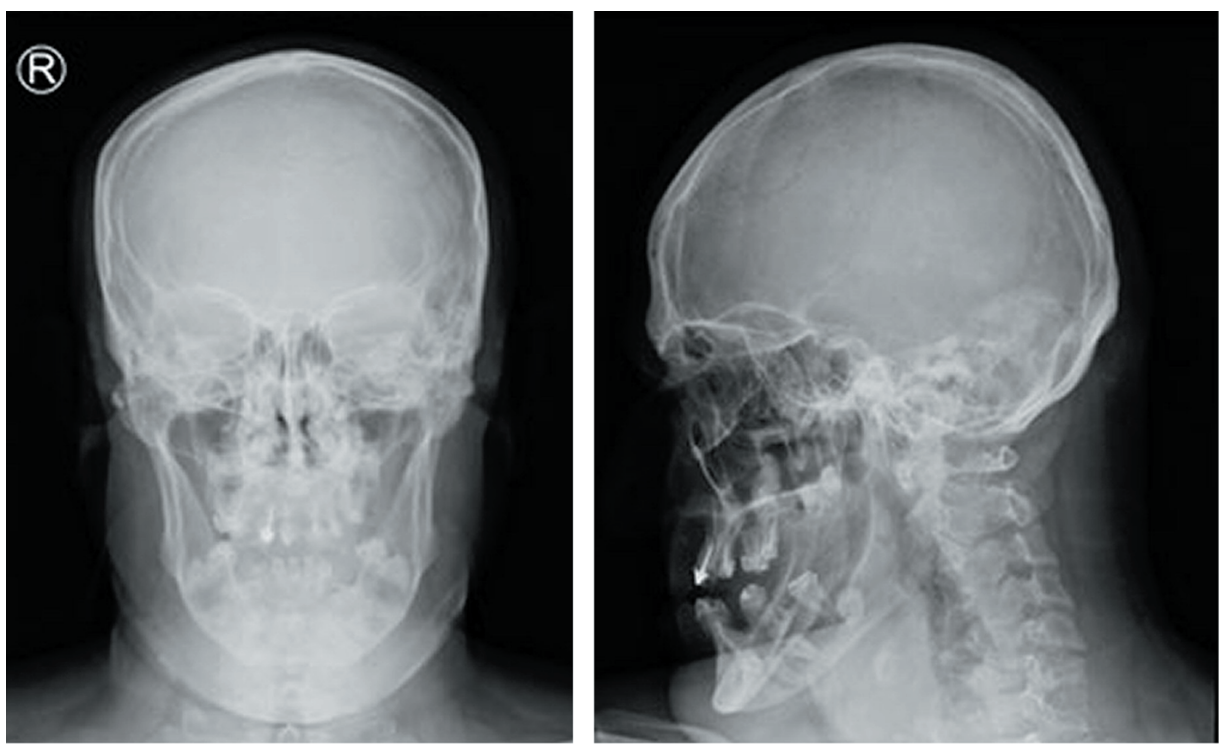

Figure 4. Skull X-ray did not reveal any other obvious deformities. 


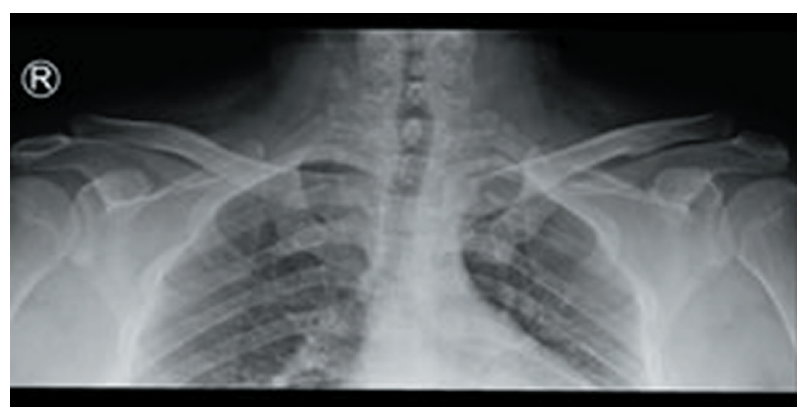

Figure 5. Chest X-ray did not reveal any other obvious deformities.

$[11,12]$. Features of Yunis-Varon syndrome are agenesis or hypoplasia of clavicle, severe micrognathia, digital anomalies, hypodontia, spinal defects, and impacted teeth. Both the syndromes presented with multiple impacted teeth but were ruled out from our case as other symptoms were not present.

In the case of hormonal disorders, hypothyroidism, hypoparathyroidism, and pseudohypoparathyroidism are to be considered.

Estimation of T3, T4, and TSH is required for diagnosing hypothyroidism where there is low serum T4 and elevated TSH. Evaluation of total serum calcium concentration and serum parathyroid hormone is required for hypoparathyroidism and pseudohypoparathyroidism. There is decrease of serum calcium in both and elevation of parathyroid hormone in pseudohypoparathyroidism and decrease of hormone in hypoparathyroidism.

Metabolic disorders like Vitamin D deficiency rickets are also associated with impacted teeth. In our case, further investigations were needed to rule out metabolic and hormonal disorders. Failure of eruption could also be due to crowding of supernumerary teeth [4], but that was not observed in our case.

Dental history and radiographic examination in this case ruled out partial anodontia. The medical and family histories along with extraoral examination were not suggestive of any syndrome or metabolic disorder.

Only a few cases of non-syndrome multiple impacted teeth were reported $[9,13]$. In those studies the predominant explanation was that some physical barrier led to impaction and non-eruption of teeth [14].

Duration and results of treatment in less frequent cases of multiple impactions is a major concern when compared to more frequent single tooth impaction cases [15]. Multidisciplinary approach would be the appropriate choice as treatment involves aesthetics, functional, and oral health problems [16]. In case of multiple impacted teeth, orthodontic extrusion may be attempted. In view of the pathological potential of the impacted teeth, surgical removal is recommended. Rehabilitation of the patient with fixed dentures needs to be planned with the help of a prosthodontist and implantologist. Obtaining stable results along with enhanced esthetics, oral health, and function would be the objective of the treatment [17].

\section{Acknowledgments}

The authors would like to thank King Saud Medical City, for providing us with the necessary tools and methods to succeed in documenting the case fully with no restrictions. We would like to also thank the patient for his willingness to contribute to the medical community in documenting his case.

\section{Competing Interest}

The authors declare that they have no competing interest.

\section{Grant Support}

None.

\section{References}

1. Kumar GS. Orban's oral histology and embryology. 12th ed. India: Mosby; 2009.

2. Wood NK, Goaz PW. Differential diagnosis of oral and maxillofacial lesions. 5th ed. St. Louis: Mosby; 2007.

3. Wise GE, Frazier-Bowers S, D'Souza RN. Cellular, molecular, and genetic determinants of tooth eruption. Crit Rev Oral Biol Med. 2002;13(4):323-334.

4. Yildirim D, Yilmaz HH, Aydin U. Multiple impacted permanent and deciduous teeth. Dentomaxillofac Radiol. 2004;33(2):133-135.

5. Andreasen JO, Pindborg JJ, Hjorting-Hansen E, Axell T. Oral health care: more than caries and periodontal disease. A survey of epidemiological studies on oral disease. Int Dent J. 1986;36(4):207-214.

6. Conley RS, Boyd SB, Legan HL, Jernigan CC, Starling C, Potts C. Treatment of a patient with multiple impacted teeth. Angle Orthod. 2007;77(4):735-741.

7. Becker A, Lustmann J, Shteyer A. Cleidocranial dysplasia: Part 1 - General principles of the orthodontic and surgical treatment modality. Am J Orthod Dentofacial Orthop. 1997;111(1):28-33.

8. Kuroda S, Yanagita T, Kyung HM, Takano-Yamamoto T. Titanium screw anchorage for traction of many impacted teeth in a patient with cleidocranial dysplasia. Am J Orthod Dentofacial Orthop. 2007;131(5):666-669.

9. Babu V, Nagesh KS, Diwakar NR. A rare case of hereditary multiple impacted normal and supernumerary teeth. J Clin Pediatr Dent. 1998;23(1):59-61.

10. Yalcin S, Gurbuzer B. Multiple impacted teeth in the maxilla. Oral Surg Oral Med Oral Pathol. 1993;76(1):130.

11. Mercuri LG, O’Neill R. Multiple impacted and supernumerary teeth in sisters. Oral Surg Oral Med Oral Pathol. 1980;50(3):293.

12. Black SL, Zallen RD. An unusual case of multiple impacted teeth. Oral Surg Oral Med Oral Pathol. 1974;37(6):975976.

13. Finkel A, Solondz G, Friedman J. Multiple supernumerary and impacted teeth. Oral Surg Oral Med Oral Pathol. 1974;37(6):976-977.

14. Bayar GR, Ortakoglu K, Sencimen M. Multiple impacted 
teeth: report of 3 cases. Eur J Dent. 2008;2(1):73-78.

15. Tanaka E, Kawazoe A, Nakamura S, Ito G, Hirose N, Tanne Y, Kawai N, et al. An adolescent patient with multiple impacted teeth. Angle Orthod. 2008;78(6):1110-1118.

16. Becker A. The orthodontic management of impacted teeth. 2nd ed. United Kingdom: Informa Healthcare; 2007.

17. Zengin AZ, Sumer AP, Karaarslan E. Impacted primary tooth and tooth agenesis: a case report of monozygotic twins. Eur J Dent. 2008;2(4):299-302. 